\title{
Holographic space and time: Emergent in what sense?
}

\author{
Tiziana Vistarini \\ University of Colorado at Boulder, United States
}

\section{A R T I C L E I N F O}

\section{Article history:}

Received 13 November 2015

Received in revised form

28 July 2016

Accepted 28 July 2016

Available online 13 September 2016

Keywords:

Holographic spacetime

Double aspect monism

Emergence

Supervenience

Duality

\begin{abstract}
A B S T R A C T
This paper proposes a metaphysics for holographic duality. In addition to the AdS/CFT correspondence I also consider the dS/CFT conjecture of duality. Both involve non-perturbative string theory and both are exact dualities. But while the AdS/CFT keeps time at the margins of the story, the dS/CFT conjecture gives to time the "space" it deserves by presenting an interesting holographic model of it. My goals in this paper can be summarized in the following way. First, I argue that the formal structure and physical content of the duality do not support the standard philosophical reading of the relation in terms of grounding. Second, I put forward a philosophical scheme mainly extrapolated from the double aspect monism theory. I read holographic duality in this framework as it seems to fit the mathematical and physical structure of the duality smoothly. Inside this framework I propose a notion of spacetime emergence alternative to those ones commonly debated in the AdS/CFT physics and philosophy circles. (c) 2016 Elsevier Ltd. All rights reserved.
\end{abstract}

When citing this paper, please use the full journal title Studies in History and Philosophy of Modern Physics

\section{Introduction}

String theory has deeply changed the traditional scientific understanding of spacetime and geometry. During the last twenty years the theory made significant progress in showing the non-fundamental nature of spacetime, whether spacetime is the geometrodynamical structure usually associated to general relativity (GR) or it is a higherdimensional spacetime with compact extra dimensions (the theory delivers different but conceptually related accounts).

However, the theory composite account of spacetime disappearance from the fundamental scale does not equally involve space and time. Indeed, the putative spacetime emergence involves primarily space. More precisely, spaces arising from lower-dimensional spaces.

This paper proposes a metaphysics for holographic duality. Inside this metaphysics I suggest a sense in which space and time may be understood as being emergent entities. The most popular instantiation of holographic duality in string theory-as far as I know the most concrete setting presently proved-is the AdS/CFT correspondence. In this paper I analyze this setting along with the $\mathrm{dS} / \mathrm{CFT}$ conjecture of duality.

Although their different conceptual status-the AdS/CFT is a proved holographic correspondence whereas the dS/CFT is a conjectured one-both involve non-perturbative string theory and both are exact correspondences. However, while the AdS/CFT keeps time at the margins of the story, the dS/CFT conjecture gives to time the "space" it deserves by presenting an interesting holographic model of it.

My attempt of delivering a metaphysics for holographic duality develops in two steps. First, I argue that the formal structures and the physical content of both correspondences do not support the philosophical interpretation of holographic duality in terms of grounding relation. Second, I put forward a conceptual scheme mainly extrapolated from the double aspect monism theory. I want to read holographic duality in this framework because the latter seems to be a conceptual category that naturally encompasses the mathematical structure and physical features of holography. Indeed the scheme provides a natural philosophical interpretation to holographic duality by delivering a metaphysical justification of its existence. Inside this framework I propose a notion of spacetime emergence along a reasoning path slightly different from that commonly used when dualities are involved. If my reading of Polchinski is correct, the second part of my philosophical proposal appears to be close to his view of dualities-see (Polchinski, pp. 29-32).

The AdS/CFT is a duality between a string theory over an Antide Sitter spacetime (the bulk spacetime containing gravity) and a conformal field theory over its boundary (not containing gravity). As far as I know this correspondence is the most concrete scenario in which the appearance of some spatial dimensions in the bulk (its radius and its compact extra dimensions) seems to be closely 
related to physical dynamics taking place in the lowerdimensional boundary (which does not contain those spatial dimensions). The prototypical case is a duality between Type IIB string theory on the spacetime $A d S_{5} \times S^{5}$ and the conformal $S U(N)$ Yang-Mills theory defined over the Minkowski boundary of this spacetime.

The dS/CFT conjecture was motivated by an attempt of finding a similar duality that could instead deliver a holographic representation of the time dimension in the bulk. The latter would be in this case a de Sitter spacetime. The appearance of the bulk's time would mimic the AdS/CFT logic underlying the appearance of the radius in the Anti-de Sitter bulk. This research program has not been taken to completion yet. Perhaps, this is why so far it did not receive the philosophical attention it deserves. Indeed this conjecture is intriguing because-if successfully tested by concrete string compactifications of the de Sitter bulk-it would involve a bulk geometry apparently more realistic than the anti-de Sitter one -which is not to say that the conjecture, at least macroscopically, is providing a realistic model of gravity. ${ }^{1}$ Moreover, it is intriguing because it also delivers a hypothetical physical equivalence between a timeless dynamical structure and a time-dependent one. In this sense, as far as I know, it is the only scenario involving string theory that manifestly undermines the fundamentality of time. Indeed the AdS/CFT does not manifestly involve time because only some spatial dimensions of the bulk become internal degrees of freedom of gauge particles in the boundary.

Section 2 contains some remarks on D-branes as they crucially contribute to the theoretical setting of both holographies. Sections 3 and 4 contain a brief, non-technical presentation of the two correspondences. Section 5 (which is divided in many subsections) puts forward the philosophical claims of this paper. I argue that holography (both AdS/CFT and dS/CFT) does not show bulk spacetime emergence from the boundary gauge theory. There isn't any plausible way of reading spacetime emergence into holographic duality. The former can be found in the latter neither as ontological notion nor as standard epistemic one. Then, what the holographic duality might be showing-if read inside the metaphysics I put forward-is an idea of what is not fundamental, namely both sides of the holographic duality and the duality itself. Section 5 is mainly divided in a pars destruens (Sections 5.1 and 5.2) and in a pars construens (Section 5.3 and all its subsections). In particular, Section 5.3.2 outlines a possible physical interpretation of the metaphysics for holographic duality I propose. ${ }^{2}$ Also, Sections 5.3.1 and 5.3.3 propose two different notions of emergence coexisting without friction in the context holographic duality, each also smoothly coexisting with the duality itself. One tries to add a dimension on the existing debate on emergence and holographic duality. The other instead tries to capture the true conceptual nature of the notion of spacetime emergence usually discussed in the AdS/CFT physics circles (i.e. usually discussed in terms of "emerging" bulk's dimensions from the boundary).

\section{Some thoughts on D-branes}

D-branes crucially appear in the AdS/CFT duality and in the dS/ CFT conjecture. These higher-dimensional objects, to which the moving open string ends attach, populate non-perturbative string

\footnotetext{
${ }^{1}$ Some evidence for a small positive value of our cosmological constant has been recently found. Also our universe is considered to have an asymptotic de Sitter structure in the future.

${ }^{2}$ Since the main goal of the present paper is that of proposing a philosophical interpretation of holographic duality, this attempt of reading the philosophical metaphor more concretely is here only sketched. The content of this section is topic for a separate paper though (see footnote 19).
}

theory's scenarios. About D-branes there appear to be two schools of thought among physicists: those thinking that D-branes undermine the exclusive primitiveness of strings in string theory, those thinking they do not. ${ }^{3}$ According to the first group strings modes would not themselves source completely D-branes dynamical features, despite those features are necessarily described by string theory. According to the second group D-branes dynamics would entirely depend on strings dynamics. ${ }^{4}$

In this section I will briefly explore this contraposition. That might seem disconnected with the philosophical goal of the paper. But this exploration has actually an important role. It is preliminary to Sections 3 and 4 that describe the basics of both holographic correspondences. The standard physics literature on which I will be relying on those two sections apparently assigns to D-branes a derived status in the physical ontology of string theory. Moreover, exploring this controversy shows to what extent establishing the correct view about D-branes' status is presently under-determined by the physical content of string theory. One might read this underdetermination as a consequence of the fact that string theory could not be the fundamental and unifying theory physicists are searching. There might be an underlying dynamical structure on which string theory's physical content may supervene. And this hypothesis (along with other hypothetical ideas) is suggested by the metaphysics for holography proposed in Section 5.

The claim that D-branes are not primitive objects in string theory is equivalent to say that if you know what all the open strings attached to a D-brane are doing, then you know what the D-brane is doing as well. In other words, from a quantum mechanical perspective, D-branes do not have degrees of freedom independent from those of open strings. That is not the same as denying that $\mathrm{D}$-branes are dynamical objects in their own right; it only means that they are not dynamically primitive in the theory. Since D-branes are derived entities, they are not put in the theory by hand: they are made out of composite strings excitations becoming visible only at strong coupling regime.

One may say against this interpretation that as a matter of fact there are Lagrangian and dynamical rules for the D-branes alone. Much like in the case of spacetime itself, it is possible to write a low-energy effective action for the D-brane. ${ }^{5}$ But such low energy action only for D-brames breaks down at high energies comparable to the string scale, as the new arising massive excitations

\footnotetext{
${ }^{3}$ In this paper primitiveness has an ontological and dynamical meaning. The two meanings are inextricably related. Strings are thought to be ontologically primitive in the physical picture delivered by the theory as their existence is established ab initio. This primitive ontological status of strings reflects the dynamical property of having physical degrees of freedom not reducible to those of other entities populating the theory's ontology. Primitive is used as opposed to derived. In this sense elementary particles are derived entities in string theory since they appear as strings modes of vibrations.

${ }^{4}$ D-branes are considered to be solitonic solutions of string theory. Broadly speaking, a soliton is any solution of some partial differential equations that is stable against decay to the "trivial solution". Solitons stability is mainly due to topological constraints, generally some boundary conditions preventing solitons from continuously deforming into the trivial solution. Solitons in string theory have the same role they play in quantum field theory: self-reinforcing, solitary waves produced by cancelation of dispersive effects in the medium. Now, in string theory these stable solutions appear to be in the closed string fields (see Taylor \& Zwiebach 2004).

5 The most popular that one can encounter in the literature is called the DiracBorn-Infeld action for arbitrary background fields-see Johnson (2003, pp. 135136):

$S_{P}=-T_{P} \int d^{p+1} \xi e^{-\Phi} \operatorname{det}^{\frac{1}{2}}\left(G_{a b}+B_{a b}+2 \pi \alpha^{\prime} F_{a b}\right)$.

As long as the curvature $G_{a b}$ is low this action describes everything at long distances regardless of the D-brane's shape. The action depends on coordinates $\xi$ representing the fluctuations of the brane transverse to the world volume. The factor of the dilaton $\Phi$ is result of the fact that all of this physics arises at open string tree level (low energy limit).
} 
living on the brane modify the dynamics (see Johnson, 2003, pp. 135-136). Physicists try to describe the changed dynamics accurately (at least to all perturbation orders of the string coupling parameter $\alpha^{\prime}$ ) by using string field theory in the D-brane world volume. The theoretical findings might suggest that quantum mechanically D-branes' degrees of freedom entirely depend on those of open strings; one might say D-branes are actually "made out" of open strings.

I think the following analogy with gravity might clarify this last point. It has been shown by some string theorists that gravity can be found in the theory by looking at some massless vibrational states of closed strings-see for example (Polchinski, 2001, pp. 108-115). So, in this sense the degrees of freedom of the GRspacetime metric tensor fail to be independent from closed string modes. ${ }^{6}$ Gravitational waves, i.e. classical perturbations of the metric tensor, are nothing beyond coherent states of closed strings in the graviton mode. D-branes, then, might be seen exactly in the same way as gravity. The classical wiggle of a D-brane would be a coherent state of open strings (Zwiebach, 2009, chap. 15).

Someone might correctly object that this hegemony of strings in the theory's primitive ontology is only apparent because it is violated as soon as we transit beyond the perturbative expansion. Because of S-duality all D-branes are equally fundamental as strings. ${ }^{7}$ But as a matter of fact there isn't any complete formalism that would make this "brane democracy" manifest. ${ }^{8}$

However, if the strings hegemony in the theory's primitive ontology will be contrasted by some new findings, there might be some conceptual difficulties that string theory will have to face in order to maintain its status of quantum gravity proposal not plagued by uncontrollable divergences. If $\mathrm{D}$-branes are not derived entities then soon or later some D-branes dynamical rules describing everything that has nothing to do with strings will be likely found. If that happens one has to face a new problem: the presumed world volume theories connected to these non-strings dynamics would have to deal with the same UV divergences as quantized gravity without strings. Strings have two-dimensional world-volumes (string world-sheets) and that is exactly what makes them so special. In a two-dimensional world-volume the possible UV divergences can be controlled. But also in a twodimensional world volume topologically non-trivial interacting histories are allowed. That does not hold true for higherdimensional world-volumes, whose UV problems are the same as those of quantized general relativity (see also Tong, 2012, section 3).

\section{AdS/CFT correspondence: the holographic nature of the bulk's radius}

This section sketches out the AdS/CFT prototypical case. Its content heavily relies on Polchinski (1995) and Zwiebach (2009). The part concerning the holographically encoded bulk radius (in the boundary dynamics) relies on two works: a paper by Balasubramanian and Kraus (1999) and a paper by de Boer, Verlinde, and Verlinde (2000). Also the background material about the correspondence mainly refers to Maldacena (1998).

The original AdS/CFT correspondence was derived by first considering two different descriptions of D-branes, one in terms of open strings and the other in terms of closed ones (see Polchinski,

\footnotetext{
${ }^{6}$ For a simple reconstruction of these physics arguments and for a philosophical use of them aimed to put forward a notion of general relativistic spacetime emergence, see Vistarini (2017, chap. 3)

${ }^{7}$ S-dualities interchange weak coupling string theory and strong coupling ones. It does that by interchanging elementary string excitations and the nonperturbative solitonic ones.

${ }^{8}$ The expression brane democracy is taken from (Taylor \& Zwiebach (2004).
}

1995). About the former, $N$ coincident D-branes at weak string coupling can be essentially seen as features of the open strings stuck to them. The number $N$ of D-branes is kept small, so gravitational effects are neglected-which amounts to say that open and closed strings are decoupled.

The massless modes of the attached open strings describe the branes completely. However, as soon as we considerably increase the number of D-branes, they turn out to be described in terms of closed strings since the gravitational effects become dominant at that point. The gravitational effects produce a curved geometry, namely the $A d S_{5} \times S_{5}$ geometry.

The two descriptions are equivalent in virtue of the open-closed dualities of string theory established in 1989 by a paper of Dai, Leigh, and Polchinski (1989). So, the key idea that led to the AdS/CFT correspondence was that there is a decoupling situation between closed and open strings in which the low energy theory of the open strings (Yang-Mills field theory propagating on the brane) is by itself equivalent to the low energy limit of the theory of closed strings propagating in the throat of the bulk (Type IIB superstring theory).

The dimensionless parameters of the Type IIB superstring theory on the bulk-i.e. the spacetime $A d S_{5} \times S_{5}$-are the string coupling $g$ and the radius $\frac{R}{\alpha^{\prime}}$ of the bulk's compact dimensions $S^{5}$ (dimensionless parameters expressed in units of the string length). Those of the $\mathrm{SU}(N)$ Yang-Mills theory are the coupling constant $g_{Y M}$ and the constant $N$ (the number of D-branes). The relations connecting the gravitational parameters to the gauge ones are the following-see (Zwiebach, 2009, pp. 541-543):

$g=\frac{1}{4 \pi} g_{Y M}^{2}$

and

$\frac{R^{4}}{\sqrt{\left(\alpha^{\prime}\right)^{2}}}=g_{Y M}^{2} N$.

By using the 't Hooft coupling $\lambda$ (Yang-Mills theory)

$\lambda=g_{\text {YM }}^{2} N$,

the two equations above can be rewritten as:

$g=\frac{\lambda}{4 \pi N}$
and
$\frac{R}{\sqrt{\alpha^{\prime}}}=\lambda^{\frac{1}{4}}$.

Although these four equations are not the formal expression of the duality, they drop a hint on it and indirectly suggest the reason for which holographic duality cannot be interpreted in terms of grounding relation. Its formal apparatus simply does not support that reading. . $^{2}$

Eq. (1)-or equivalently (3)-shows that the weak Yang-Mills coupling is directly proportional to the weak string coupling. One may think that by sticking to a weak coupling regime, it is possible to make both theories simultaneously tractable, hence making the AdS/CFT testable. But the direct proportionality between $R$ and $\lambda$ in (4) reveals that things are not so simple. Indeed, in order to have tractability of the gauge theory we need weak coupling and a small t'Hooft parameter $\lambda$, whereas to have tractability of the gravitational theory we still need to have weak coupling, but associated with a large $\lambda$, since the radius $R$ of $S^{5}$ has to be large (to keep the curvature small).

As I said, the lack of simultaneous tractability is an obstacle to testing the correspondence. However, assuming the latter is

\footnotetext{
${ }^{9}$ A precise technical presentation of how the physical parameters on each side of the duality relate to each other can be also found in Polchinski (p. 30).
} 
correct, the four identities just mentioned show a sort of complementarity between the two sides: those effects that make impossible to make computation in a theory, make it possible to compute things in the other one (Zwiebach, 2009, p. 543).

I am not explicitly reconstructing in this paper the complete formal articulation of the AdS/CFT correspondence. It suffices to mention that the formal apparatus of the correspondence is a one-to-one map in the spectrum of the two theories, with equality of observables. Broadly speaking, observables in CFT (expectation values of products of local operators) get mapped by the duality to observables of the string theory (basically perturbations of boundary conditions).

Why is this correspondence considered to be holographic? To answer this question I will unravel some important aspects of the covariance energy-radius. Strictly speaking, this particular interplay between the boundary and bulk dynamics does not explicitly show up in the formal expression of the duality. But it is an important physical feature of the correspondence that sheds more light on what "holographic" means in this context.

The broad idea is that some boundary data of the dualitydescribing a gauge field theory-encode the radial evolution of bulk fields by controlling the appearance of a bulk radius. The radial dimension of the bulk is encoded in the boundary fields data in the form of energy variation. Physical events taking place in the bulk at great distance from the boundary are encoded in the boundary in the form of IR processes, whereas events taking place at small distances from the boundary are encoded in UV boundary processes. So, the radial dimension of the bulk where strings live "is" an internal degree of freedom of gauge particles living in the boundary, which does not contain that radial dimension.

Parenthetically, boundary data also holographically control the appearance of compact extra dimensions in the bulk, but in this paper I will only consider the holographic reconstruction of the bulk's radius. This is the crucial feature that the AdS/CFT duality shares with the dS/CFT conjecture. As I said, the conjecture, by mimicking the logic underlying the AdS/CFT duality, attempts to deliver a holographic representation of time.

The standard methodology starts from the classical action of the gravity theory in the bulk by computing the classical solution with boundary values for the bulk's field and for the bulk's metric (see de Boer et al., 2012, also see Balasubramanian \& Kraus, 1999). Then, broadly speaking, the action evaluated on this solution (along with some other technical steps not mentioned here for simplicity reasons) shows to be in a one-to-one correspondence with the generating function of (gauge invariant) operators in the gauge field theory. As I just said, this shows that a process in the bulk not far away from the boundary, amounts to keeping a finite UV cut-off in the gauge theory, whereas at great radial distances from the boundary the correspondence shows IR boundary dynamics in the gauge theory.

A simplified version of the covariance radius-energy is the following: let's call $\phi$ the bulk field solution of the gravity action with fixed boundary values; let $\phi_{\text {boun }}$ denote the boundary field related to the bulk field by those fixed boundary values; finally let's use $U(\phi)$ to denote the potential of the bulk field $\phi$ propagating throughout the bulk. Bulk and boundary fields get to know each other over the boundary. The simplified version of their covariance looks like:

$\beta\left(\phi_{\text {boun }}\right)=\frac{[\ldots] \partial U(\phi)}{U(\phi)}$,

where on the left-hand side $\beta\left(\phi_{\text {boun }}\right)$ denotes the renormalization group (RG) flow of the boundary field, namely the beta functions describing the change of the coupling of the boundary field with respect to the change of the energy scale taking place over the boundary. The right-hand side contains information about the radial evolution of the bulk field coupling with closed strings and other bulk fields; the quantity within the square parenthesis (omitted for simplicity reasons) refers to the couplings of the bulk field.

As I said, the identity (5) is a simplified version of the energyradius covariance. Strictly speaking it is not the canonical expression of the AdS/CFT, but it gives an idea of why the AdS/CFT is called holographic. And it also recalls the symmetrical structure of the correspondence. The identity (5) shows that the RG flow of the boundary field theory is in a one-to-one correspondence with the variation of the potential of the scalar field radially propagating in the bulk.

There is a bridge between the boundary gauge field theory and the bulk string theory "connecting" their physical contents. A quite popular metaphor of this inter-theoretical relation refers to the existence of a dictionary translating the language of either sides into the language of the other. Through this dictionary the variation of energy in the boundary get translated into variation of radial distances. The formal structure of this physical covariance does not support the idea that one of the two sides of the duality may be grounding the other.

Is there any similar correspondence in which holography applies to time rather than to space? As I said there is an interesting conjecture about the holographic nature of time. It describes some time-dependent bulk physics as an internal degree of freedom of some dynamics playing themselves out over the boundary.

\section{4. dS/CFT conjecture: towards a holographic description of time}

An inquiry about a possible holographic nature of time requires theories with asymptotic structures different from those of the AdS/ CFT case. Indeed the bulk spacetime involved in the conjecture is De Sitter. Such spacetime-differently from the AdS one-has a boundary at timelike infinity. Broadly speaking, it has been thought that if a theory of quantum gravity exists in a de Sitter spacetime, then its hypothetical dual would be a timeless quantum field theory over the spacetime boundary. Such a theory would have the same formal structure as that of a quantum field theory on a spacelike surface.

So far any attempt to fully achieve a quantum gravity theory in a de Sitter spacetime failed. Indeed, several attempts of embedding de Sitter spacetime as a solution of string theory did not produce satisfactory results. The absence of concrete string compactifications of de Sitter spacetime makes hard formulating and testing a dS/CFT conjecture in the string context.

A first attempt of conjecturing a dS/CFT correspondence is made by Strominger (2001)-along with Balasubramanian, de Boer, and Minic (2003). Their conjecture in principle does not require string theory inputs. The key idea is that a hypothetical correspondence should satisfy at least two requirements in order to mimic the AdS/CFT duality. First, symmetries of the dual theories must match through the correspondence. Second, it must provide a dictionary for translating correlation functions. As we saw in the previous case, the second requirement is the source for developing the holographic correspondence between the boundary fields behavior (with respect to the change of energy scale) and the dynamical fields evolution in the bulk (radial evolution in the AdS/CFT and time evolution in the dS/CFT).

If my reading is correct, Strominger, while looking for a dS/CFT map satisfying the first requirement, explicitly computed the asymptotic symmetric group of the de Sitter. ${ }^{10}$ The topological structure of this group indicates that if a quantum gravity theory in a de Sitter exists, then its hypothetical dual would be a local quantum conformal field theory on the Euclidean boundary at time $t= \pm \infty$.

\footnotetext{
${ }^{10}$ The Euclidean conformal group in D dimensions-SO(D,1) if one thinks of the de Sitter as a hyperboloid embedded in flat spacetime of signature $(1, \mathrm{D})$.
} 
Moreover, they showed-working on the second requirement-that the trace of the stress boundary tensor-which has a natural relation with the RG flow of the field theory-provides information on how the bulk spacetime reacts to changes of the boundary metric. In particular they obtained a sort of formal relation between dilatations over the boundary and time translation in the bulk. Here I won't give any technical details. This program has not taken into completion yet and the formal details necessary to a complete and precise reconstruction would be indeed outside the scope of this paper. The list of findings just mentioned is indeed enough informative about the nature of the project and its parallels with the AdS/CFT case. ${ }^{11}$

Summarizing, about 2001, the mentioned authors obtained the holographic conjecture dS/CFT without any inputs from string theory. The conjecture tells you how a string theory in de Sitter spacetime would look like, if it exists.

A later attempt of embedding the conjecture dS/CFT in the context of string theory is developed by Gutperle and Strominger (2003) and it uses D-p branes and S-p branes. D-p branes are Dbranes in $\mathrm{p}+1$ dimensions, whereas $\mathrm{S}$-p branes are thought to be the spacelike version of ordinary D branes. Perhaps better, they are branes in which every tangential dimension is spacelike. They have $\mathrm{p}+1$ dimensions, all spatial.

Briefly sketching the authors reasoning, they argue thatalthough we cannot directly obtain from string theory the dS side of the conjecture-the theory would produce the timeless Euclidean field theory required by the CFT side.

The crucial step was reached when they showed that string theory contains the above mentioned space-like versions of Dbranes as result of decay of unstable D-p branes. Unstable D-p branes are D-branes with a tachyon field whose potential resembles a double well. As such D-branes decay, they disappear completely, leaving behind a background of closed string propagating in a time dependent geometry.

The hope was that, in analogy with the AdS/CFT duality where an "emergent" spatial dimension of the bulk arises from some physics taking place in the branes making up the boundary, these space-like branes would lead to an "emergent" time direction in the bulk. ${ }^{12}$ Now, as I said, string theory admits these spacelike branes among its solutions. And the Euclidean field theory required by the dS/CFT seems to be naturally related to these branes. ${ }^{13}$

\section{Emergent space, emergent time, but in what sense?}

Inside the philosophical debate holographic duality is often used as a sort of theoretical finding that grounds the notion of spacetime emergence. The standard approach is that of thinking the bulk spacetime (along with gravity) as of an emergent structure arising from the dual lower dimensional gauge theory on the boundary (see, e.g. Horowitz \& Polchinski, 2006; Rickles, 2012; Seiberg, 2007;).

\footnotetext{
${ }^{11}$ For technical details I refer the reader to the papers mentioned above in this section.

${ }^{12}$ Formally speaking, these S-branes are time-like kink solitonic solution. A kink solution in string theory is defined in the same way as it is presented in ordinary $\phi^{4}$ theory: it is the solution to a double well potential $U(\phi)=\left(\phi^{2}-a^{2}\right)$, with vacua $\phi_{ \pm}= \pm a$. Roughly speaking at weak coupling this is a very localized lump of energy.At strong coupling it is a sharply localized particle. So, in this sense $\phi$ is an interpolating solution between the two vacua. Being time-like in this context means that $\phi \rightarrow \pm a$ when the time coordinate, radial than the spatial one, goes to infinity (Srednicki, 2007).

${ }^{13}$ The authors computed some gravity solutions proved to be dual to the "timeless" quantum field theory. What has been found so far are: (1) D = 4 Einstein-Maxwell gravity corresponding to a timeless theory on a charged So brane; (2) the bosonic action of $D=11$ supergravity theory corresponding to a timeless theory over an S-5 brane.
}

Before looking closer at the philosophical landscape, let's summarize what it means to say that the two theories are holographically dual. Although the boundary and bulk theories are different ${ }^{14}$ they share the same physical content. In other words, the two theories make precisely the same predictions about all observable phenomena. The duality relates their respective physical observables. The expected values of any observable in any state are the same in both theories (see also Rickles, 2011). Another way to put it is that the holographic duality is a symmetrical relation between the boundary theory and the bulk one, a one-to-one correspondence inextricably relating boundary dynamics with bulk ones.

The holographic nature of the AdS/CFT correspondence is commonly traced in the fact that the radius of the bulk "lives" in the boundary as internal degree of freedom. More precisely, the radius appears to be "the same as" the renormalization group flow of the boundary (the radius is not a spatial dimension of the boundary). It is usually claimed that the bulk radius "emerge" from the boundary dynamics. In the case of the dS/CFT conjecture the same logic is tentatively implemented, but here two different theories relate to each other by virtue of holography. What it is thought to be "emergent" from the boundary is the time dimension of the bulk: time arising from timeless boundary dynamics.

In this section I want to criticize the idea that the holographic duality (AdS/CFT and dS/CFT) can be used to ground spacetime emergence. The symmetric mathematical and physical character of holography does not account for the asymmetrical nature of emergence-something on the same line is developed by Teh (2013) and by Rickles (2012).

One might object that my claim about the asymmetrical character of emergence holds true as long as I connect emergence to fundamentality issues; indeed it might pointed that I am implicitly using an ontological notion of emergence and that this is not by all means necessary. One might be looking for a weaker asymmetry. Some notion not involving fundamentality but still accounting for a novelty. Perhaps, some kind of epistemic emergence of the spacetime structure from its dual partner. My answer is that-as I will argue in Section 5.2-the standard philosophical notion of epistemic emergentism also does not seem to fit the context of holography.

So far I have been sketching the pars destruens of my paper which is developed in Sections 5.1 and 5.2. The paper's pars construens is instead developed in Section 5.3 (and its subsections). The latter is a philosophical interpretation of holography in a framework mainly extrapolated from the double aspect monism approach. Inside this scheme I will propose a picture in which two different notions of emergence coexist.

\subsection{Holographic duality and supervenience-based emergence}

A paper by Dean Rickles analyzes to what extent it is possible to read the AdS/CFT duality as a case of spacetime emergence:

"If we are to speak of the emergence of spacetime in the philosophers'sense then it must be the case that spatiotemporal physics is supervening on non-spatiotemporal physics. In other words, we ought to find that spacetime geometry [is] not part of some 'deeper' theory, but arise as novel consequence [...]. A weaker option, still along the same lines is that one kind of spacetime geometry supervenes on another different kind of spacetime geometry or structure. The standard claim within AdS/CFT circles is that the emergent structure is a space with gravity $[\ldots]$. So clearly the sense in which space is emerging here corresponds to my weaker option[...]: quantum gravity is derivable from gauge theory."(Rickles, 2012, pp. 316-317).

\footnotetext{
${ }^{14}$ They refer to different spacetimes, they have different formal apparatuses and so on. More on this difference will be said in the last two sub-sections.
} 
In this section I argue that the duality between the gauge theory and the gravity one (both AdS and dS) cannot be interpreted in terms of standard supervenience-based notion of emergence.

Let's see what the standard philosophical view of emergence in terms of supervenience amounts to. It is generally called "supervenience emergentism" (see O'Connor \& Wong, Hong Yu, 2015). ${ }^{15}$ It was suggested by McLaughlin in 1997: "If $\mathbf{P}$ is a property of $\mathbf{w}$, then $\mathbf{P}$ is emergent if and only if (1) $\mathbf{P}$ supervenes with nomological necessity, but not with logical necessity, on properties the parts of w have taken separately or in other combinations; and (2) some of the supervenience principles linking properties of the parts of $\mathbf{w}$ withw's having P are fundamental laws" (McLaughlin, 1997, p. 39).

Part (2) of this definition reveals why it is so hard to keep together emergence via supervenience and holographic duality. More precisely, it shows why it is problematic to claim that one side of the duality supervenes the other one. Indeed, part (2) says that in order to establish if a physical property of a structure is emergent we first need to have an independent criterion of fundamentality.

This characterization of emergence is for example smoothly applicable to all those cases in which the presumed emergent property arises via a mechanism involving a change of energy scale (or length scale). The derivation of general relativity solutions by reimposing conformal invariance at the quantum string level is one of these cases. The change of energy scale (or length scale) is formally encoded in the derivation of general relativity spacetime, so in this sense the derivation itself already has an asymmetric formal structure necessary to the grounding. Then a metaphysical interpretation of this formal asymmetry is the bottom-up approach to fundamentality: about the planck scale physical dynamics are more fundamental than those taking place at larger scales. ${ }^{16}$ In other words, the formal asymmetry of the derivation, along with a metaphysical interpretation (the bottom-up approach) of this asymmetry, provide a criterion of fundamentality which is then used to establish if a property is emergent. Note that the derivation of general relativity from quantum string theory does not involve duality. The two theories are not physically equivalent.

Now, in the case of holographic duality, nothing in its formal structure shows some trace of asymmetry. One element of the dual pair can be derived from the other and vice versa. Nothing in the formal covariance of the physical parameters on each side of the duality shows uneven levels of fundamentality. So, holographic duality does not seem to fit supervenience-based emergence.

A further concern about the use of supervenience arises from considering one of its most peculiar features. Before appearing in the debate on spacetime emergence, supervenience was proposed in philosophy of mind to build a token-physicalism-something that could avoid the type-type identity of mind and brain without incurring ontological emergentism of mind. To this aim its most distinctive feature is that of accounting for multiple realizability. This characterization of supervenience-which is aimed to violate the one-to-one relation-is usually considered to be somehow canonical. This does not hold true only in broad philosophy of science, but also in the more restricted philosophy of physics circle. But applying this canonical notion of supervenience in the context of holographic duality produces problems. Multiple realizability conflicts with the nature of duality which is instead a one-to-one correspondence-one configuration of the radial (temporal) evolution of the bulk's field is "realized" in the boundary by only one configuration of the RG flow.

\footnotetext{
15 See O'Connor, \& Wong, (2015, par. 3.1).

16 This case-that I call general relativistic spacetime emergence-can be seen as a case of emergence via supervenience (see Vistarini, 2017, chap. 3): the formal derivation itself, by virtue of its asymmetric structure, provides an unambiguous criterion of fundamentality.
}

Now, if the next move is that of taking multiple realizability out of supervenience, we definitely re-gain the one-to-one correlation, but then in what sense we keep talking about supervenience-based emergence of one side from the other? In the presence of a one-toone correlation all it is left seems to be a type-type identity, in other words emergence dissolves to leave room to reduction. As concluding remark, however we conceptually manipulate superveniencebased emergence in order to avoid ontological emergentism, we cannot get rid of the asymmetry (symmetric supervenience is simply not supervenience). And the derivation of a theory from its dual partner can be hardly seen as containing such asymmetry.

\subsection{Holographic duality and epistemic emergence}

The deflationary notions of emergence delivered by the philosophical standard debate on epistemic emergence do not seem to fit the inter-theoretical relation instantiated by holographic duality. Roughly speaking, the many philosophical versions of epistemic emergence existing in the current philosophical landscape are divided in two broad groups (see O'Connor, Wong, 2015). They both offer an account of weak emergence that bypasses the strong asymmetry described in the previous section. However, each provides a form of epistemic irreducibility that can hardly be applied to holographic duality.

In the first group, called the predictive approach, all the views seem to share an idea: an emergent property of a complex system arises as organization of its parts which is not predictable by looking to the way in which each single part functions. For example, the unpredictable emergent property can be a non-linear organization of the system's parts. Emergent properties cannot be predicted from the knowledge of the pre-emergent stage of the system, of the laws ruling its parts'behavior.

This summary does not definitely account for the many subtleties characterizing this group of views. For example I am not differentiating here the diachronic unpredictability from the synchronic one. Indeed it is the latter that more or less brings in a notion of epistemic irreducibility, namely that of the whole with respect to its parts.

However, the summary seems sufficient to show that trying to attach this label of epistemic emergence to the holographic duality is a nonstarter. Complex systems play a crucial role, and the epistemic emergence is defined within the relation of irreducibility they have with their own parts. This picture seems to be very different from a scenario in which the presumed emergent spacetime does not seem to arise from its own parts, rather from a dual gauge structure.

The second group shares instead the use of a different idea of epistemic irreducibility, namely that of irreducible pattern. ${ }^{17}$ Typically the emergent properties are considered to be features of systems ruled by lawlike generalization inside the domain of some special science (for the original source of this approach see Fodor, 1974; see O'Connor and Wong, 2015 for some recent review). The emergent properties appear in higher-level descriptions and are irreducible to properties populating lower-level descriptions: for example, the attempt of deriving principles of psychology directly from principles of quantum mechanics inevitably fails since psychological patterns cannot be captured (at least not entirely) by the formal language and physical content of quantum mechanics.

Here the asymmetric nature of the inter-theoretical relation-i. e. the emergence of psychological patterns from underlying quantum dynamics, along with a form of pattern-irreducibility not readable in the other way around-is mainly due to the uneven degree of fundamentality owned by the theories' physical

\footnotetext{
${ }^{17}$ In the actual debate the distinction between the first and the second group is not so sharp and net as it appears here. As I said, inside the first group unpredictability is used in some case to evoke an idea of irreducibility, although the notion acquires different nuances in that context.
} 
domains. Either domain is defined by a physical length scale of size quite different from that of the other and the quantum size is considered to be the most fundamental between the two.

Now, one may attempt to apply such notion of irreducible pattern to those physical theories whose physical parameters relate to length scales mutually very close-i.e. physical length scales that can provide a rough estimate of each other. ${ }^{18}$ The AdS bulk theory and the gauge boundary theory would be a pair of exactly this kind (see Polchinski, p. 30)-eq uivalently the dS bulk theory and its dual gauge boundary.

By restricting our attention to pairs of physical theories both defined over short spatial scales, we manage to leave out of the comparative picture the strong asymmetry induced by the large unevenness of physical sizes. Then we may try to trace this second idea of epistemic irreducibility in the holographic relation between the boundary and the bulk theories.

But what we find in the holographic relation is an unambiguous formal bridge between the two theories through which dynamical patterns on one side completely captures dynamical patterns on the other side. The theories' physical content is not lost in translation, not even partially.

One may correctly argue that irreducibility in the holographic case has to be found by looking at those features of the dual theories with respect to which the duality is blind. In other words, features like different geometry, different number of spacetime dimensions, a timeless geometry versus a time-dependent one, and so on. All these things are not enclosed in the set of physical observables of the two theories. But still these things crucially contribute to produce a picture of irreducibility.

This argumentative strategy is separately analyzed in Sections 5.3.1 and 5.3.2 as it delivers a notion of irreducibility fitting holographic duality. However this idea of irreducibility does not actually fit the conceptual scenario delivered by the second epistemic approach we are considering here. Indeed, according to this standard approach, irreducibility seems always to touch the physical content of the theories involved in the comparison (at least partially). Now, if we want to stick to this standard interpretative framework, we have to claim that the type of irreducibility arising in holography somehow touches the physical content of the two theories in question-something left out of the duality, yet physically salient. Then, given the covariance among their physical parameters and the mutual closeness of their length scales, the two theories might appear to be competitors rather than dual.

\subsection{Holographic duality and spacetime emergence reconciled}

The AdS/CFT duality is commonly presented in the philosophical debate as a case of emergence of space from a lower-dimensional gauge physics, whereas the $\mathrm{dS} / \mathrm{CFT}$ conjecture of duality (so far on the edge of the main stream of philosophical discussion) would be a case of time emergence from a lower-dimensional and timeless gauge physics. So far I argued that interpreting both dualities in terms of grounding relations between a gauge field theory (grounding) and a spacetime/gravity theory (grounded) does not seem to produce a plausible view. I argued that duality because of its formal and physical features cannot be used to implement both strong and weak spacetime emergence (respectively, supervenience-based emergence and epistemic emergentism).

In this section I propose a metaphysics for holographic duality in terms of a philosophical scheme mainly extrapolated from the double-aspect monism approach. Then, I will show that this

\footnotetext{
${ }^{18}$ The Planck scale is the natural first guess for a rough estimate of the string length scale.
}

metaphysical framework can be combined without friction with a specific line of reasoning leading to spacetime emergence.

The double aspect theory has a long and illustrious tradition (see Stubenberg, 2014). From Spinoza (1677) to Strawson (1959). Then, more recently from Nagel (1986) to Chalmers (1996). All these views seem to share a broad metaphysical assumption that can be expressed by the following general statement: the multiple, different and apparently unrelated existence of phenomena might be "explained" by-or "reduced" to-the existence of a unique "structure" (or "substance") manifesting itself through multiple and different aspects (or "modes"). Broadly speaking, each aspect is real and distinct. Each amounts to be the "structure" manifesting itself through a specific pattern. The aspects can be two (for example the double aspect monism approach to the mind-body problem) or finitely many more, or even infinitely many more (like in Spinoza's view).

Each of these views has its own account of the precise conceptual nature of the aspect-structure relation. I will consider the bare bones of this relation shared by all these pre-existing frameworks and I will use these basic facts in my discussion on holography. This will require me to specify what the aspects and the "structure" are in my case. Leaving aside for a moment this specification, here I only make the preliminary claim that the relation aspect-structure is specified as supervenience. But this supervenience is not read into (or put on top of) the holographic duality, as the supervenience-based approach to spacetime emergence does. Rather the holographic duality itself is among the things that supervene on some underlying structure. Also, in what follows I present neither the history of double aspect monism nor some of its versions in depth.

\subsubsection{Holographic duality read through a conceptual metaphor}

The goal of this section is to deliver a philosophical analysis of holographic duality that smoothly fits its mathematical structure and physical content. The framework of the analysis is extrapolated from some features of double aspect monism.

The picture of reality consistent with double aspect monism imposes necessarily two general constraints on all the possible ways in which the two aspects can mutually relate. The first is that either aspect is different from the other. In this sense the relation between the two aspects can be read in terms of irreducibility. The second constraint is that there is a neat one-to-one correspondence between their different components, despite the two aspects are overall mutually irreducible.

More specifically, how would irreducibility (possibly evoking an idea of mismatch) coexist with the one-to-one structure of the matching between the two aspects? We may think that the two aspects are made of the same set of individual components, or units, but each aspect corresponds to a different collective arrangement of these units. Both aspects would be two distinct outcomes arising from two different patterns of organization of the same set of individual units. This interpretation fits the central metaphysical assumption of any variant of double aspect monism, i.e. the idea that the two aspects are distinct manifestations of the same reality. What I am adding to this minimal assumption is the explicit characterization of this reality in terms of being a structure "underlying" the two aspects.

In what follows this framework will be also called the conceptual scheme (or just scheme).

The structure of the holographic duality between the gauge field theory and the gravity theory (presented in section three and four) seems to be a mathematical and physical instantiation of this conceptual scheme. In other words the conceptual scheme appears to be a natural context of philosophical interpretation for holographic duality, a context apparently more appropriate than those philosophical interpretations previously mentioned.

On the one hand the scheme is different from those interpretations of holographic duality in terms of emergence via supervenience. The latter has indeed the problem to reconcile its 
asymmetric structure-naturally brought into the story by the notion of supervenience-with the symmetrical character of the duality. To this aim, any attempt to weaken supervenience turns out to dissolve the notion. On the contrary the one-to-one mapping structure of the holographic duality smoothly finds a natural philosophical counterpart in the second constraint of the scheme. So, in this sense the dual match between the gauge theory and the gravity one is metaphysically justified by the framework: the holographic correspondence is one-to-one because the two theories are two aspects of the same underlying fundamental physical content.

On the other hand, the scheme is different from the philosophical framework of epistemic emergence. Earlier I argued that the latter carries a notion of epistemic irreducibility that can hardly fit the kind of irreducibility arising between the two dual sides of holography. This irreducibility seems more or less due to some form of blindness of the holographic duality towards some features of the two dual theories. So, in this sense, from the perspective of either theory, those features of the other which are left out the mapping are irreducible. But the features neglected by the duality are not physical observables in both dual theories. So the irreducibility does not seem to involve the physical contents of the two dual theories, hence it co-exists with the theories' physical equivalence.

On the contrary, the epistemic irreducibility carried by the standard epistemic approach seems to require some (at least partial) incomparability between physical contents of the theories which are being compared. That does not happen with the scheme. Indeed in the latter the idea of irreducibility and that of one-to-one correspondence (broad conceptual denotation for physical equivalence) are both necessary conditions of the same metaphysical assumption. So in this sense they can logically coexist without friction.

Inside the conceptual scheme, the gauge field theory on one side of the duality and the gravity theory on the other side, would be both different descriptions (aspects) of the same fundamental physical reality. The latter would be a sort of underlying tertium quid manifesting itself in two physically equivalent ways, still very different. Let's leave aside for a moment any further considerations about the nature of this difference between the two sides of the duality. We will come back to this in the next section.

Reading the holographic duality in this scheme highlights an important feature of the duality. Far from being a grounding relation, it still provides an unambiguous criterion to establish what is not physically fundamental. Each theory of the holographic dual pair is not fundamental as long as it is conceived as some arising pattern of organization of more fundamental physical degrees of freedoms, still unknown. The gauge field theory and the gravity one are both non-fundamental in equal way, in the sense that they do not ground each other.

\subsubsection{The conceptual metaphor in more concrete terms}

As I said, the primary goal of this paper is that of proposing a philosophical metaphor that can smoothly interpret the physical content of holography without relying on hidden metaphysical assumptions external to that content. Indeed the scheme philosophically characterizes the dual relation by simply sticking to the bare formalism of holography.

As also Dieks, Dongen and de Haro argue, the symmetrical nature of the correspondence cannot ground an uneven level of fundamentality between its two sides. The authors interestingly characterize this symmetrical properties in terms of "exactness" of the duality (see Dieks, Dongen, \& de Haro , 2015, pp. 13-15).

However, this section does not want to add philosophical considerations about the metaphor I propose. Rather, it wants to outline briefly some more concrete thoughts about the latter, though still related to the main philosophical stream of the paper. ${ }^{19}$ This section is an attempt of imagining a possible physical scenario that may concretely instantiate the philosophical metaphor of double aspect monism proposed so far.

The idea that the gravity and gauge theories are two mutually irreducible manifestations of the same underlying physical structure is the main metaphysical assumption of these philosophical approach. This assumption metaphysically justifies the covariance of physical patterns occurring separately on each side. And a metaphysical justification usually suggests a specific theoretical framework in which physical findings can be interpreted. If the framework turns out to be empirically adequate then the metaphysical assumption crucially contributes to define the physical meaning of those findings.

But now the question is: what kind of physical mechanism might be suggested to physically explain this covariance in terms of double aspect monism?

The physical ontology of the presumed underlying theory might be populated by physical entities which are more fundamental than particles and strings (populating the two dual theories). Their fundamentality might be described by the fact that such physical units combine together to create the complex physical scenario delivered by the duality. Their more fundamental degrees of freedom may give rise somehow to the less fundamental degrees of freedom of the dual pair (parenthetically, to see how the counting of the latter match between the AdS side and the CFT one see Zwiebach, 2009, chap. 15).

More precisely, every physical state on each side of the duality might be thought as supervening some underlying pattern of combination of these fundamental units. But in this scenario we don't have only a set of physical states on each side. We also have a relation of covariance among them. And this covariance can also be interpreted as a physical relation supervening some specific underlying relations among fundamental entities. One may argue that these underlying relations are entangled states. So, the entangled states of the presumed underlying quantum field theory would control the higher-level covariance formally showed by the holographic duality.

The covariance is holographic because it shows some features peculiar of holograms: what represents an external degree of freedom (space or time) in the bulk is an internal degree of freedom of some dynamics in the boundary. But differently from a real hologram we cannot find in this case an uneven degree of fundamentality between the two representations. This intertwined appearances of the two different physical scenarios, if read into the double aspect monism can be seen as a physical property supervening entangled states of underlying physical structures. A way of finding a basic identikit of the subvenient basis might be that of individuating its physical degrees of freedom. The latter, still unknown, could be somehow hidden in the presently available dynamical equations characterizing the physical laws governing the two dual scenarios.

How does spacetime emergence come into the story? The next section will try to show how. As I said holographic duality relates observables of either theory with those of the other. But holographic duality does not relate features that are outside the physical contents of the two theories. Those who are neglected contribute to the diversity of the two dual theories. They also entirely account for irreducibility. And spacetime is one of those neglected features.

\footnotetext{
${ }^{19}$ This outline is one of the topics analyzed in a separate, technical paper I am writing about quantum entanglement and spacetime, in particular about those findings showing that quantum entanglement might serve the thread that stitches spacetime together. In my paper physical and philosophical implications of this idea are developed in light of the paper by Maldacena \& Susskind (2013). In their paper the authors suggest possible resolutions of the firewalls paradoxes by interpreting a special class of GR solutions-those in which two distant black holes' interiors are connected through a wormhole-as maximally entangled states forming complex EPR pairs.
} 


\subsubsection{Conclusion: spacetime emergence in what sense?}

As we saw, by reading holographic duality inside the conceptual scheme we gain an unambiguous criterion to establish what is not fundamental. According to the scheme both dual theories are not fundamental. And their duality relation is not fundamental as well, since it would formally represent a presumed physical relation supervening entangled states of underlying dynamics.

The two dual theories are physically equivalent but, as I said, their physical contents "look" different. For example, an observable in the conformal field theory side is the expectation value of some local operators, whereas an observable in the gravitational side is a perturbation of some boundary condition. Also, in the conformal field theory side there are field states which are mapped onto string states in the gravity side, and so on.

As I said, these differences produce a notion of irreducibility that cannot be applied to the physical contents of the two theories because the duality's dictionary (formally identifying observables of each side and field states with graviton strings states) establishes the reduction in two ways.

The hypothesis presented in the previous sections propose to describe this peculiar kind of diversity in terms of two hypothetical facts. First, the two scenarios are different because they may supervene different dynamical states (states predicted by the tertium underlying theory). Moreover, the two scenarios are dual (physically equivalent) because the underlying dynamics they each supervene may be entangled. So, in this sense their duality relation would also gain a physical meaning, namely that of being a relation supervening that physical entanglement (this meaning would be formally reflected in the dictionary of translation).

Now, from the point of view of each theory of the holographic dual pair, the physical content of the other does not appear emergent because of the existence of the formal dictionary establishing the identity bridge between the two physical contents.

Emergence via supervenience can be only traced in the vertical relation between the dual pair of theories and the underlying complex network of entangled states (predicted by the presumed tertium theory). Very importantly, this emergence via supervenience should not be identified and confused with the notion of spacetime emergence as traditionally discussed in the AdS/CFT circles-i.e. the emergence of extra bulk's dimensions from the lower dimensional boundary.

To include in the picture this notion of spacetime emergence in a way that capture its true philosophical status, we need to shift our attention from the physical content of the two theories-in other words from the domain of application of the holographic duality-to those features of the two theories neglected by the holographic correspondence.

Holographic duality is apparently blind to properties like spacetime structure, number of dimensions and so on. There is no dictionary for translating them from one side to the other. As I anticipated in Section 5.2, a structure of either theory that does not get translated by the holographic duality in some structure of the other, is an irreducible property of the theory. This irreducibility contributes to the overall diversity of the two theories, but without breaking their physical equivalence. More importantly, this irreducibility is correlated to an idea of spacetime emergence not in tension with the symmetrical nature of holographic duality.

The spacetime structures of the bulk and of the boundary (in both AdS/CFT and dS/CFT) are untouched by the holographic duality. From the theoretical perspective of the boundary theory the overall AdS spacetime is a structure not reducible to anything in its own description of the world. Therefore, it is emergent in that description. And the same holds in the other way around by assuming the bulk's theoretical perspective.

Summarizing, I used those theoretical features living outside the domain of application of dualities to define a notion of irreducibility. This notion is compatible with the physical equivalence of the two dual theories. This notion in turn brings in a definition of spacetime emergence that is still an epistemic kind of notion with a weak asymmetry only readable in one way each time (although overall it goes in two ways). Indeed the weak asymmetry in the case of the bulk spacetime emergence is grounded on choosing the perspective of the boundary theory as the privileged (or preferred) one. The weak asymmetry can be read in the other way by changing the metaphysical assumption and picking the bulk's perspective as the preferred one.

If my reading of Polchinski is correct, the way in which he suggests to think about spacetime/gravity emergence in the setting of gauge/gravity duality seems to have some conceptual closeness with my proposal.

Gauge/gravity duality, starting from QFT side is an example of an emergent gravity.[...] We discussed emergent gauge theory, so emergent gravity may not be such a surprise."("Dualities" by J. Polchinski, Studies in History and Philosophy of Modern Physics, 2015, p. 32)

This passage shows an appealing internal articulation. The notion of emergence in "emergent gauge theory" may be understood in light of his previous discussion in the paper about dualities among quantum field theories. In this theoretical setting he also refers to the fact that dualities only relate physical observables. As he says, gauge structures are not physical observables. So, they do not get translated through the duality's dictionary. So, Polchinski says, the gauge invariance of either side of the duality would appear to be emergent from the perspective of the other side; in other words it would appear to come out of nothing.

This looks quite close to my analysis of spacetime emergence. However, his use of the expression "emergent gravity" might be interpreted in two ways. If he is saying that gravity would emerge from a quantum field theory underlying both gravity and its gauge dual partner, then his proposal goes along with mine. If instead what he means is that gravity would emerge from the dual field theory, then this is exactly one of the points I reject in my paper.

\section{References}

Balasubramanian, V., de Boer, J., \& Minic, D. (2003). Exploring De Sitter Space and Holography. Annals of Physics, 303(1), 59-116 [hep-th/0207245].

Balasubramanian, V., \& Kraus, P. (1999). Space-Time and the Holographic Renormalization Group. Physical ReviewLetters, 83, 3605 [hep-th/9903190].

Chalmers, D. (1996). The Conscious Mind. Oxford: Oxford University Press.

Dai, J., Leigh, R. G., \& Polchinski, J. (1989). New Connections between String Theories. Modern Physics Letters A, 4, 2073-2083.

de Boer, J., Verlinde, E. P., \& Verlinde, H. L. (2000). On the Holographic Renormalization Group. Journal of High Energy Physics, 0008, 003 [arXiv:hep-th/991]

Dieks, D., van Dongen, J., \& de Haro, S. (2015). Emergence in holographic scenarios for gravity. Studies in History and Philosophy of Modern Physics, 52(Part B), 203-216 [arXiv:hep-th/1501.04278v2].

Fodor, J. (1974). Special Sciences. Synthese, 28, 91-115.

Gutperle, M., \& Strominger, A. (2002). Spacelike Branes. Journal of High Energy Physics (p. 2002), 2002. http://dx.doi.org/10.1088/1126-6708/2002/04/018.

Horowitz, G. T., \& Polchinski, J. (2006). Gauge/Gravity Duality In: D. Oriti (Ed.) Approaches to Quantum Gravity. Toward a New Understanding of Space, Time and Matter (pp. 169-186). Cambridge: Cambridge University Press [arXiv:gr-qc/ 0602037].

Huggett, N., \& Vistarini, T. (2015). Deriving General Relativity from String Theory Philosophy of Science, 82, 1163.

Johnson, C. V. (2003). D-Branes. Cambridge: Cambridge University Press.

Maldacena, J. M. (1998). The large N limit of superconformal field theories and supergravity. Advances in Theoretical and Mathematical Physics, 2, 231-252 [International Journal of Theoretical Physics, 38, 1113 (1999)] arXiv:hep-th/ 9711200.

Maldacena, J. M., \& Susskind, L. (2013). Cool horizons for entangled black holes Fortschritte der Physik, 61(9), 781-811. http://dx.doi.org/10.1002/ prop.201300020.

McLaughlin, B. (1997). Emergence and Supervenience. Intellectica, 2, 25-43.

Nagel, T. (1986). The View from Nowhere. Oxford: Oxford University Press. 
O'Connor, T., \& Wong, H. Y. (2015). Emergent Properties. In: The Stanford Encyclopedia of Philosophy. 〈http://plato.stanford.edu/entries/properties-emergent/〉.

Polchinski, J. (1995). Dirichlet Branes and Ramond-Ramond Charges. Physical Review Letter, 75, 4724 [hep-th/9510017].

Polchinski, J. (2001). String Theory Volume 1. Superstring Theory and Beyond. Cambridge: Cambridge University Press.

Polchinski, J. (2016). Dualities. Studies in History and Philosophy of Modern Physics. pp 29-32.

Rickles, D. (2011). A Philosopher looks at String Dualities. Studies in History and Philosophy of Modern Physics, 42, 54-67.

Rickles, D. (2012). AdS/CFT Duality and the Emergence of Spacetime. Studies in History and Philosophy of Modern Physics, 44, 312-320.

Seiberg, N. (2007). Emergent Spacetime In: D. Gross, M. Henneaux, \& A. Sevrin (Eds.), The Quantum Structure of Space and Time (pp. 163-178). World Scientific Publishing.

Spinoza, B. (1677). Ethics. The Collected Writings of Spinoza (2 vols), vol. 1. Princeton (NJ): Princeton University Press.
Strawson, P. F. (1959). Individuals. London: Methuen.

Strominger, A. (2001). The dS/CFT correspondence. Journal of High Energy Physics, 0110, 034 [hep-th/0106113].

Stubenberg, L. (2014). Neutral Monism, in: The Stanford Encyclopedia of Philosophy. 〈http://plato.stanford.edu/archives/fall2014/entries/neutral-monism/〉.

Taylor, W., \& Zwiebach, B. (2004). D-Branes, Tachyons and String Field Theory. Lectures presented at TASI 2001. [arXiv:hep-th/0311017v3].

Teh, N. (2013). Holography and Emergence. Studies in History and Philosophy of Modern Physics, 44(3), 300-311.

Tong, D. (2012). Lectures on String Theory. [arXiv:hep-th/09080333v3].

Vistarini, T. (2017). The Emergence of Spacetime in String Theory. Routledge forthcoming.

Zwiebach, B. (2009). A First Course in String Theory (2nd edition). Cambridge: Cambridge University Press. 